\title{
Global Drug Repurposing Research from 2000 to 2018: A Bibliometric Analysis
}

\author{
Wei $\mathrm{Li}^{1,2,3, ~ *}$, Li Wan ${ }^{1,2,3}$ \\ ${ }^{1}$ Department of Pharmacy, Maternal and Child Health Hospital of Hubei Province, Wuhan, China \\ ${ }^{2}$ Women and Children's Hospital of Hubei Province, Wuhan, China \\ ${ }^{3}$ Maternal and Child Health Hospital of Hubei Province, Tongji Medical College, Huazhong University of Science and Technology, Wuhan, \\ China
}

\author{
Email address: \\ liwei1988hust@126.com (Wei Li) \\ ${ }^{*}$ Corresponding author
}

\section{To cite this article:}

Wei Li, Li Wan. Global Drug Repurposing Research from 2000 to 2018: A Bibliometric Analysis. Advances in Bioscience and Bioengineering. Vol. 9, No. 2, 2021, pp. 39-47. doi: 10.11648/j.abb.20210902.14

Received: May 13, 2021; Accepted: May 24, 2021; Published: June 3, 2021

\begin{abstract}
Objectives: Drug repurposing (DR), a promising tool to accelerate drug discovery, has increasingly drawn scientists' attention. This study aims to explore the landscape of DR through visualization analysis on DR-related publications. Methods: Data were collected from the Web of Science Core Collection between 2000 and 2018. Data was analyzed in terms of publication outputs, journals, countries, institutions, authors, co-authorship, co-citation, research hotspots and evolution trends through VOSviewer and CiteSpace. Results: From 2000 to 2018, we can see a continuous growth in the number of DR related publications. PLoS One published the highest number of publications, followed by Scientific Reports, Oncotarget and Antimicrobial Agents and Chemotherapy. The United States (USA) was the most leading country in DR research, in terms of publications, institutions, co-authorship and co-citation. Talevi A (Argentina) was the most productive authors and Ashburn TT (USA) contributed the most cited articles in this area. The hotspots of DR research were cancer, drug discovery, apoptosis, polypharmacology and virtual screening, whereas the emerging trends of DR were connectivity map, database, pathway and apoptosis. Conclusions: This study might help beginners to quickly understand the cutting-edge knowledge, mainstream research directions, hot spots and research trends of this research field.
\end{abstract}

Keywords: Drug Repurposing, Bibliometric Analysis, VOSviewer, CiteSpace, Drug Discovery

\section{Introduction}

Drug repurposing (DR) is defined as exploiting novel indications for existing drugs beyond its original uses or initial approved indications [1]. In fact, $30 \%$ of the FDA approved drugs were derived from repurposed drugs [2]. Recent years, drug repurposing has aroused the attention of drug companies, as the increasing high costs and disappointing success rate of drug discovery process [3]. Repurposing can markedly lower the cost and shorten the development cycle, because of the definite pharmacokinetic and safety data of existing drugs [4]. Sildenafil, is the world-wide known example of drug repurposing, which was originally developed for angina pectoris treatment but, due to continual erections adverse event, was repurposed for erectile dysfunction [5]. Although a large number of DR studies have been published on academic journals, few efforts have been made to analyze the evolution of scientific outputs in this field systematically. Bibliometric analysis has been widespreadly used in various fields to assess previous research activity, track evolution process and explore research hotspots and frontiers [6-11]. Herein, bibliometric analysis was employed to obtain the quantitative and visual information in the global scientific outputs of DR research from 2000 to 2018, identify its pivotal points and emerging trends.

\section{Materials and Methods}

\subsection{Data Collection}

Data were downloaded from the Web of Science Core 
Collection (WoSCC). Online retrieval was conducted in October 11, 2019 by using key terms "drug repurposing" OR "drug repositioning" OR "drug reprofiling" OR "drug redirecting" OR "drug rediscovering". Time span was set to between 2000 and 2018. The publication types were limited to article or review or proceedings paper or letter and languages were to English.

\subsection{Data Extraction}

The query retrieved 3,220 bibliographic records between 2000 and 2018 on October 11, 2019. Chose the full record and cited references option, when downloading data from WoSCC. Tab-delimited file format is recommended for VOSviewer, while plain text file format is for CiteSpace. The bibliographic records, including title, abstract, authors, journals, institutions, countries, keywords, references, WoSCC categories and impact factor (IF) of the journal were extracted.

\subsection{Data Analysis}

VOSviewer 1.6.13 was used to analyze the journal citation, co-authorship and co-citation network. CiteSpace 5.4 was performed to analyze the co-citation keywords. OriginPro 9.1 was applied to make histograms and line charts. In network visualization, each node represented an item (journal, institution, keyword, etc.), and the size of nodes represented the frequency of occurrence. The distance between two nodes represented the relevance of two items. Nodes adopting different colors in network represented different clusters.

\section{Results}

\subsection{Analysis of Publications}

From 2000 to 2018 , a total of 3,220 publications in DR research were found. As shown in Figure 1, the annual number of publications continually increased over time. The accelerated increase in DR research literature appeared in 2010. The number of publications in 2018 (705 articles) and 2014 (275 articles) was more than 14-fold and 5-fold higher than that in 2010 (50 articles), respectively.

The 3,220 publications in DR research were published in 1,143 academic journals. The top 15 active journals were presented in Table 1. PLoS One [impact factor (IF) 2018, 2.776] published the highest number of articles (102 articles, $3.16 \%$ ), followed by Scientific Reports (IF2018, 4.011; 82 articles, 2.55\%), Oncotarget (IF2016, 5.168; 73 articles, 2.27\%), Antimicrobial Agents and Chemotherapy (IF2018, 4.715; 46 articles, 1.43\%), Drug Discovery Today (IF2018, $6.880 ; 43$ articles, $1.34 \%$ ). The top 15 academic journals account for $20.16 \%$ of the total publications on DR research (649 articles). Compared with other journals, articles in DR study were more potentially to be accepted by these active journals.

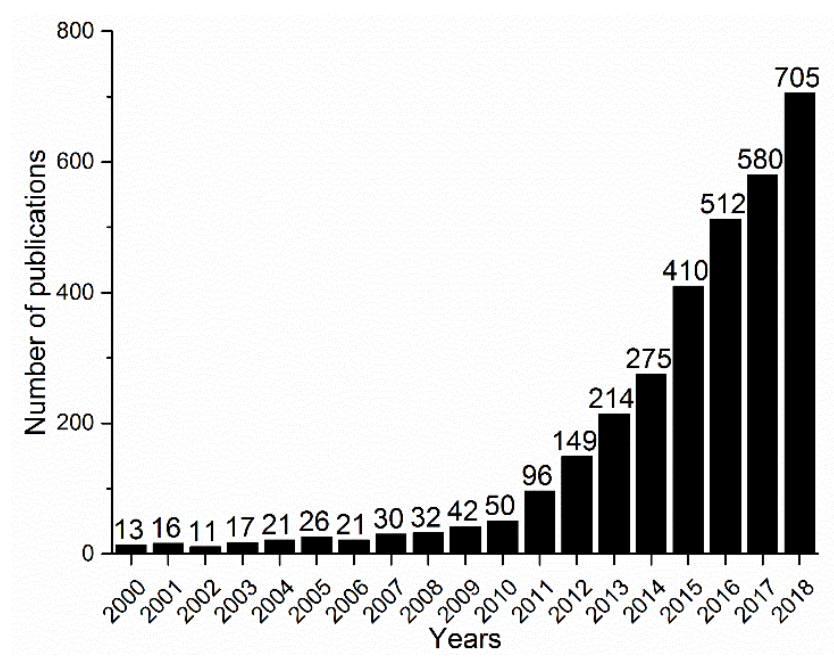

Figure 1. Distribution of yearly publications on DR research.

Table 1. Top 15 active journals from 2000 to 2018.

\begin{tabular}{|c|c|c|c|c|c|c|}
\hline Rank & Journal & Country & N1 (\%) & $\mathbf{N 2}$ & N1/N2 & IF2018 \\
\hline 1 & PLoS One & USA & $102(3.16)$ & 208,242 & 0.05 & 2.776 \\
\hline 2 & Sci Rep & UK & $82(2.55)$ & 83,100 & 0.10 & 4.011 \\
\hline 3 & Oncotarget & USA & $73(2.27)$ & 21,961 & 0.33 & $5.168^{*}$ \\
\hline 4 & Antimicrob Agents Chemother & USA & $46(1.43)$ & 16,596 & 0.28 & 4.715 \\
\hline 5 & Drug Discov Today & UK & $43(1.34)$ & 4,146 & 1.04 & 6.880 \\
\hline 6 & BMC Bioinformatics & UK & $39(1.21)$ & 9,123 & 0.43 & 2.511 \\
\hline 7 & Curr Top Med Chem & Netherlands & $36(1.12)$ & 3,083 & 1.17 & 3.442 \\
\hline 9 & Assay Drug Dev Technol & USA & $32(0.99)$ & 886 & 3.61 & 1.420 \\
\hline 10 & J Chem Inf Model & USA & $29(0.90)$ & 3,691 & 0.78 & 3.966 \\
\hline 11 & J Med Chem & USA & $29(0.90)$ & 14,289 & 0.20 & 6.054 \\
\hline 12 & Proc Natl Acad Sci USA & USA & $27(0.84)$ & 70,885 & 0.04 & 9.580 \\
\hline 13 & Front Pharmacol & Switzerland & $26(0.81)$ & 4,111 & 0.63 & 3.845 \\
\hline 14 & Curr Pharm Des & Netherlands & $25(0.78)$ & 7,827 & 0.32 & 2.412 \\
\hline 15 & Expert Opin Drug Discov & UK & $24(0.75)$ & 1,289 & 1.86 & 4.421 \\
\hline
\end{tabular}

Notes: N1 (\%), total number of DR related articles in a journal from 2000 to 20018; N2, cumulative number of articles in a journal from 2000 to 2018, N1/N2, the ratio of the total number of drug repurposing related articles to the cumulative number of articles from 2000 to 2018 ; 2018 IF, Journal Impact Factor in 2018; IF= impact factor, USA=United States of America, UK= United Kingdom; *, 2016 IF.

Furthermore, journal citation analysis was adopted to reflect the quality of a literature [12]. In this network, the 
activity of journal was described by the size of nodes and the link strength between 2 nodes was illustrated by the distance between 2 nodes. A shorter distance indicated a stronger relation and a higher citation frequency. Nodes with the same color belonged to a cluster. As shown in Figure 2, journals were divided into 5 clusters. The red cluster consisting of Oncotarget, Cancer research, Cancer letters, Molecular cancer therapeutics, Drug discovery today, representing journals in cancer related research. The green cluster consisting of PLos One, Molecular biosystems, BMC bioinformatics, BMC system biology, Journal of biomedical informatics, representing journals in genomics and bioinformatics. The blue cluster consisting of Scientific Reports, Antimicrobial agents and chemotherapy, Journal of virology, Current pharmaceutical design,
Frontier in microbiology, representing journals in microorganism related research. The yellow cluster consisting of European journal of medicinal chemistry, Bioorganic \& medicinal chemistry, Future medicinal chemistry, Current topics in medicinal chemistry, Molecules, representing journals in chemistry. The violet cluster consisting of Biochemical pharmacology, British journal of pharmacology, Expert opinion on drug discovery, Combinatorial chemistry \& high throughput screening, representing journals in pharmacology and fundamental research. Most of the top active 15 journals in Table 1 were also shown in Figure 2, the higher IF, the larger node, revealing that journals with higher IF had more citations.

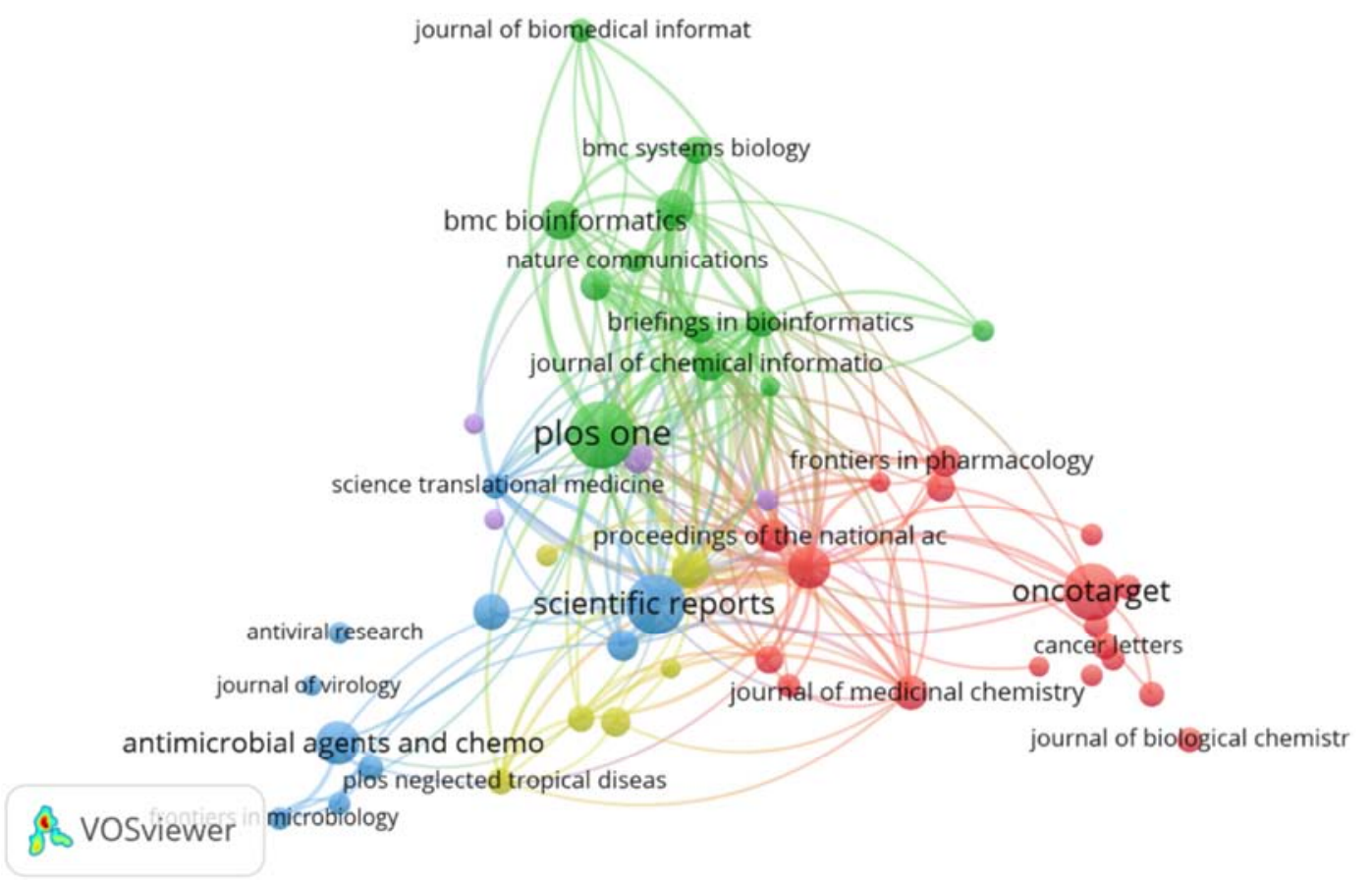

Figure 2. Journal citation analysis network.

Table 2. 10 leading countries and institutions that published DR related articles from 2000 to 2018 .

\begin{tabular}{lllll}
\hline Rank & Country & Frequency & Institution (Country) & Frequency \\
\hline 1 & USA & 1,344 & Chinese Academy of Sciences (China) & 52 \\
2 & China & 384 & Harvard University (USA) & 40 \\
3 & UK & 339 & Stanford University (USA) & 38 \\
4 & Italy & 222 & University of Cambridge (UK) & 35 \\
5 & Germany & 211 & Case Western Reserve University (USA) & 34 \\
6 & India & 186 & Harvard Medical School (USA) & 33 \\
7 & Canada & 134 & University of Toronto (Canada) & 32 \\
8 & South Korea & 121 & National Institutes of Health (USA) & 31 \\
9 & France & 114 & University of California at San Francisco (USA) & 30 \\
10 & Japan & 112 & University College London (UK) & 30 \\
\hline
\end{tabular}

\subsection{Country and Institution Co-authorship Analysis}

The 3,220 DR research articles were contributed by 91 countries/territories. The top 10 countries (4 European countries, 4 Asian countries and 2 American countries) devoted 3,167 articles, accounting for $98.35 \%$ of the total publications (Table 2). The USA (1344 articles, 41.74\%), China (384 articles, 11.93\%) and UK (339 articles, 10.53\%) were the top three countries. As shown in Figure 3, the USA had cooperative relationships with 56 countries and was the most cooperative country. Its cooperative countries mainly include China, Canada, Germany, UK, Italy and India. 
3,332 institutions participated in the study of DR. Chinese Academy of Sciences ranked the first place, followed by Harvard University and Stanford University. The top 10 institutions contributed 355 articles, accounting for $11.02 \%$ of the total publications (Table 2).

Institution co-authorship analysis was performed to reflect the degree of communication between institutions and the influential institutions in this field. Institutions were ranked by the number of citations of their published articles. The institution co-authorship network with 98 nodes (top 98 influential institutions) was showed in Figure 4. The Harvard University (USA, 2,560 citations), the Massachusetts General Hospital (USA, 1,664 citations) and the Stanford University (USA, 1,336 citations) are the top 3 influential institutes with the largest number of citations in DR related publications.

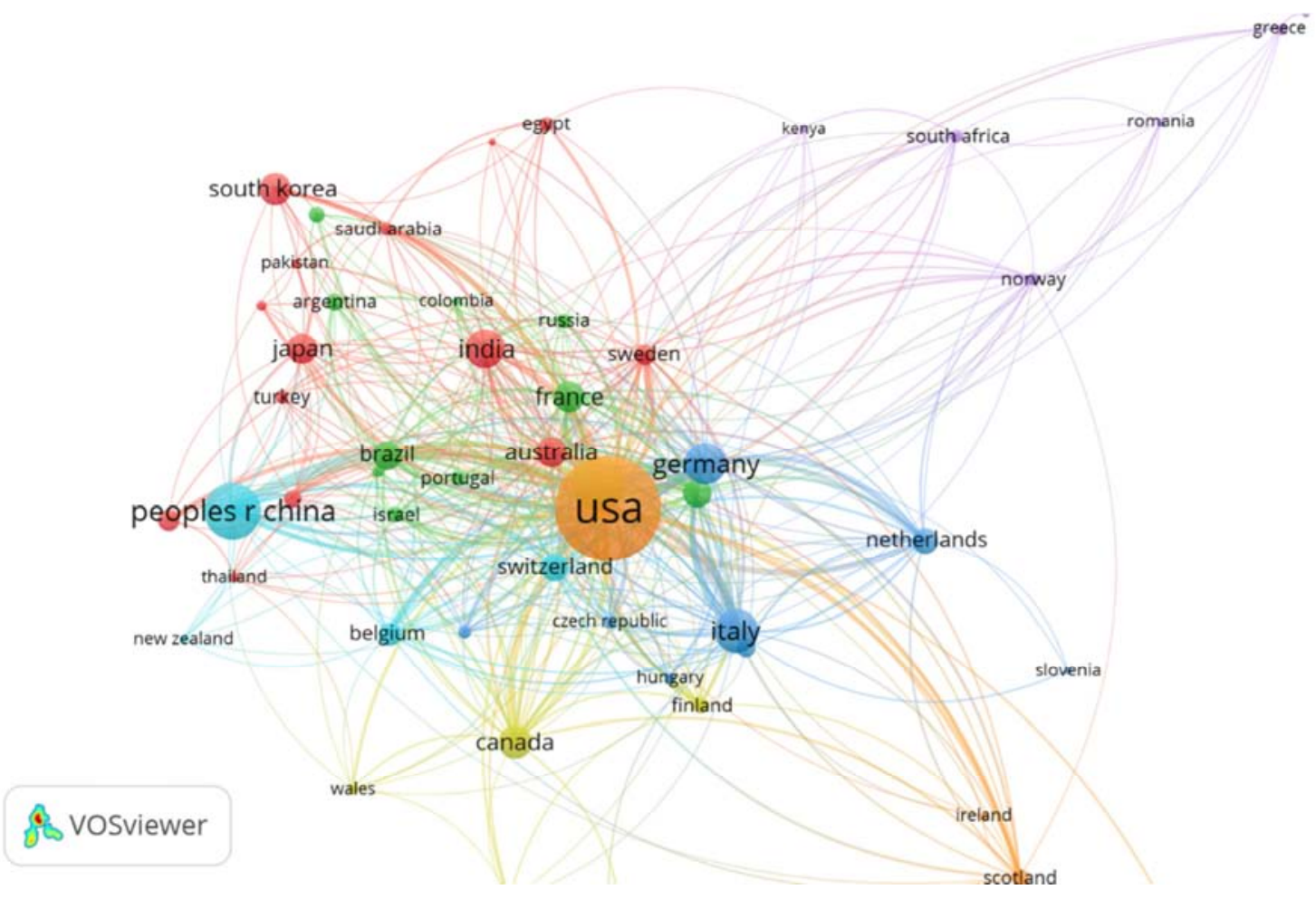

Figure 3. Country co-authorship network.

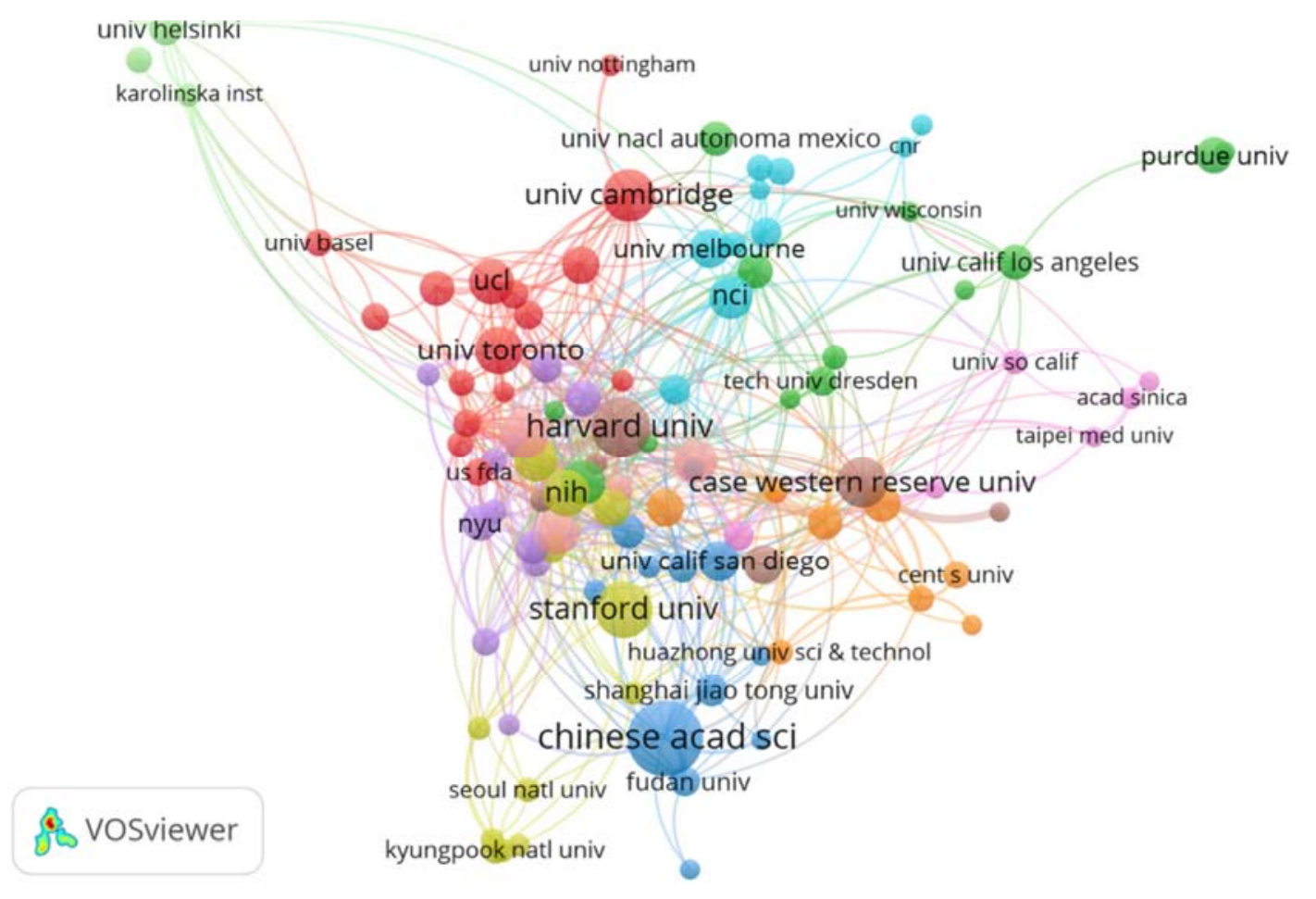

Figure 4. Institution co-authorship network. 


\subsection{Author Co-authorship Analysis}

These 3,220 publications were drafted by 15,976 authors. The top 20 prolific authors ranked by number of publications were listed in Table 3. Talevi A (Argentina, 18 articles) took the lead, followed by Mucke HAM (Austria, 16 articles), Xu R (USA, 16 articles) and Pollastri MP (USA, 15 articles). The top 20 prolific authors contributed 241 article $(7.48 \%$ of the total) to DR research. Of the 20 productive authors, 11 authors came from the USA. However, the average number of citations (avg. citations) of articles of these authors was not consistent with the rank of the publication number, indicating these authors should pay more attention to the quality and rather than the quantity of their articles. As shown in Table 3, Butte AJ (11 articles, 1175 citations) ranks first with an avg. citations of 106.82, followed by Dudley JT (74.35) and Tang Y (64.22), revealing their important role in DR research. Butte AJ (USA) cooperates with Dudley JT (USA), Sirota M (USA), Roedder S (USA) and Sarwal MM (USA). Talevi A (Argentina) with the most publications, cooperates with Carrillo C (Argentina), Bellera CL (Argentina), Balcazar DE (Argentina) and BrunoBlanch LE (Argentina). Pollastri MP (USA, Northeastern University), Zheng W (USA, NIH), Austin CP (USA, NIH) and Cheng FX (USA, Northeastern University) have many collaborators. (see in Figure 5).

Table 3. Top 20 prolific authors ranked by number of publications

\begin{tabular}{|c|c|c|c|}
\hline Author (Country) & Frequency (citation) & Author (Country) & Frequency(citation) \\
\hline Talevi A (Argentina) & $18(232)$ & Butte AJ (USA) & $11(1,175)$ \\
\hline Mucke HAM (Austria) & $16(16)$ & Xie L (USA) & $11(561)$ \\
\hline Xu R (USA) & $16(176)$ & Duenas-Gonzalez A (Mexico) & $10(206)$ \\
\hline Pollastri MP (USA) & $15(336)$ & Pantziarka P (UK) & $10(129)$ \\
\hline Cheng FX (USA) & $14(672)$ & Sukhatme VP (USA) & $10(254)$ \\
\hline Dudley JT (USA) & $14(1,041)$ & Zhao ZM (USA) & $10(128)$ \\
\hline Seleem MN (USA) & $13(269)$ & Bouche G (Belgium) & $9(195)$ \\
\hline Wang QQ (USA) & $13(154)$ & Schroeder M (Germany) & $9(253)$ \\
\hline Andre N (France) & $12(351)$ & Tang Y (China) & $9(578)$ \\
\hline Zheng W (USA) & $12(296)$ & Wang Y (China) & $9(230)$ \\
\hline
\end{tabular}

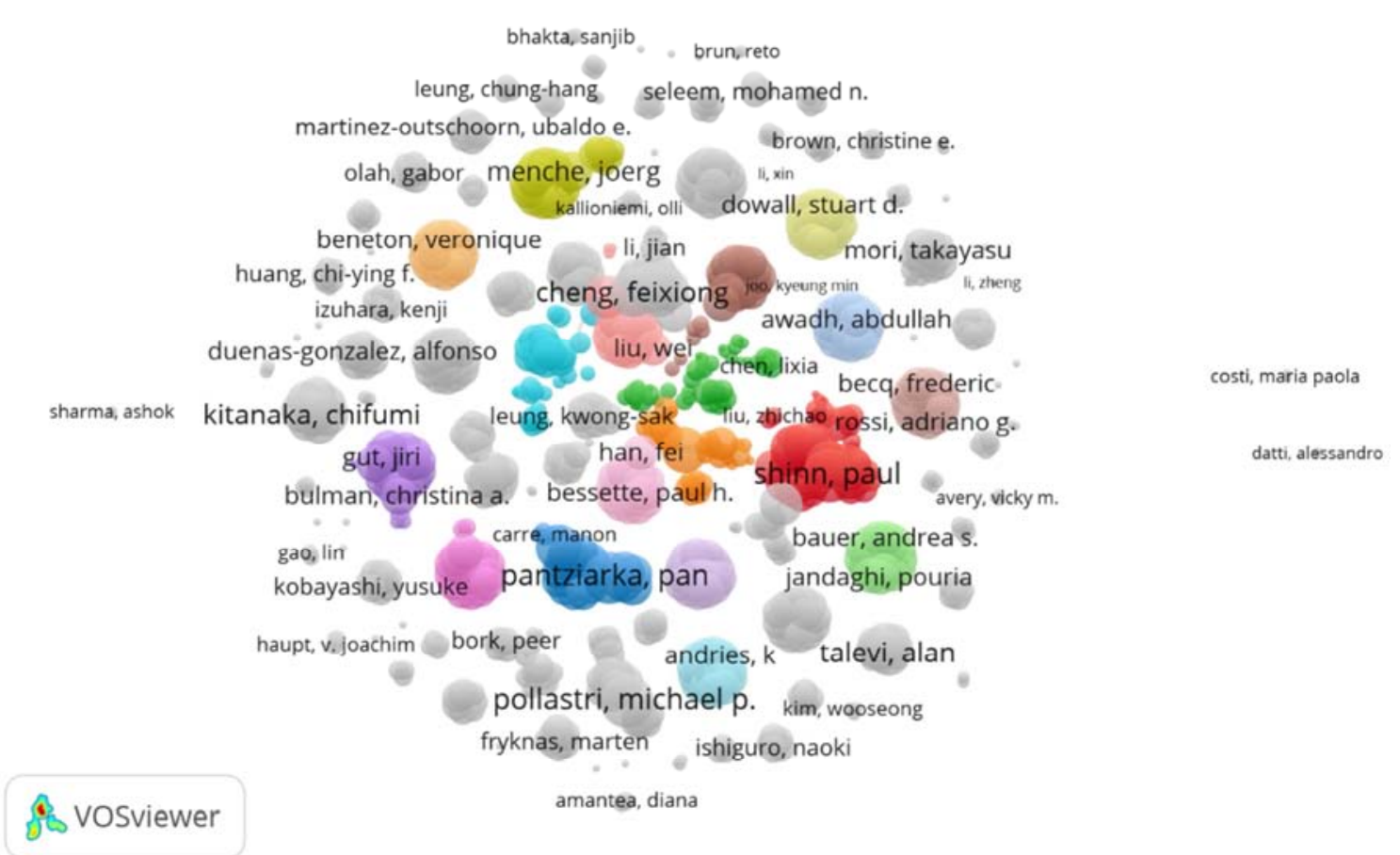

Figure 5. Author co-authorship network.

\subsection{Cited Authors and References Co-citation Analysis}

A co-citation relationship between two items (references, journals or authors) was established when simultaneously cited in a third citing item's reference list [13]. As shown in Figure 6 , the top 543 authors (ranked by citation number) were presented in the cited author co-citation network. Ashburn TT (454 citations) ranked the first, followed by
Lamb J (374 citations), Keiser MJ (348 citations), Wishart DS (318 citations), Dudley JT (291 citations), Iorio F (270 citations), Cheng FX (257 citations), Kuhn M (239 citations), Chong CR (234 citations) and Hopkins AL (232 citations). The biggest node was Ashburn TT et al [3], indicating that his important role in DR research. Ashburn TT et al systematically reviewed DR in Nature Reviews Drug Discovery. In Figure 6, authors who studied in a similar field 
and had close cooperation with each other were assigned into one of the six colored clusters.

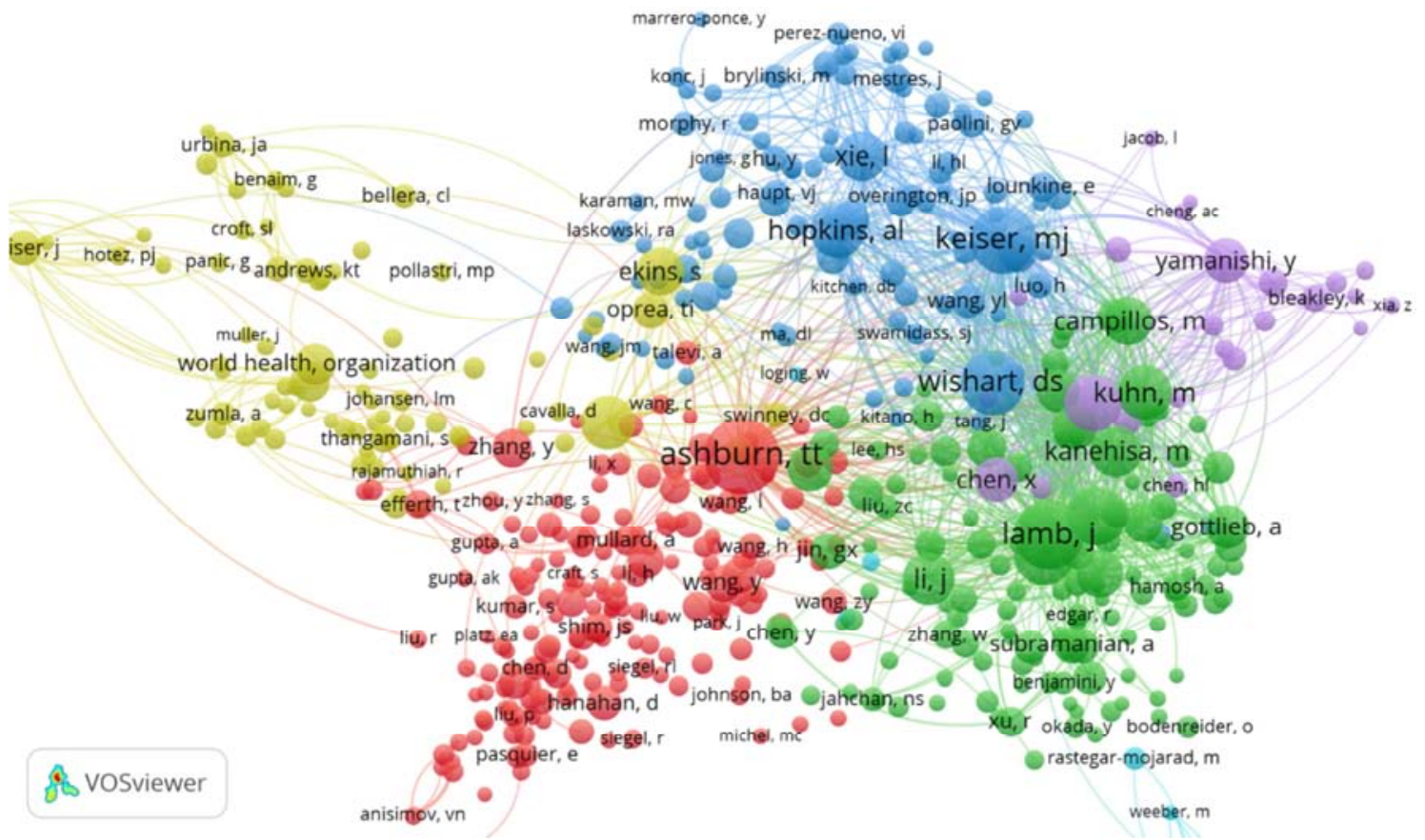

Figure 6. Cited author co-citation network.

Here, VOSviewer 1.6.13 was applied to construct the cited reference co-cited network, including 247 nodes (top 247 cited references) and 17,467 links. The size of the nodes revealed the number of its citations and their association with DR research. For instance, as exhibited in Table 4 and Figure 7, the largest node was the article by Ashburn TT et al, titled
"Drug repositioning: identifying and developing new uses for existing drugs", which was published in Nature Reviews Drug Discovery (2004). This article was cited 454 times altogether in these 3,220 DR related publications and was cited 1,010 times in WoSCC.

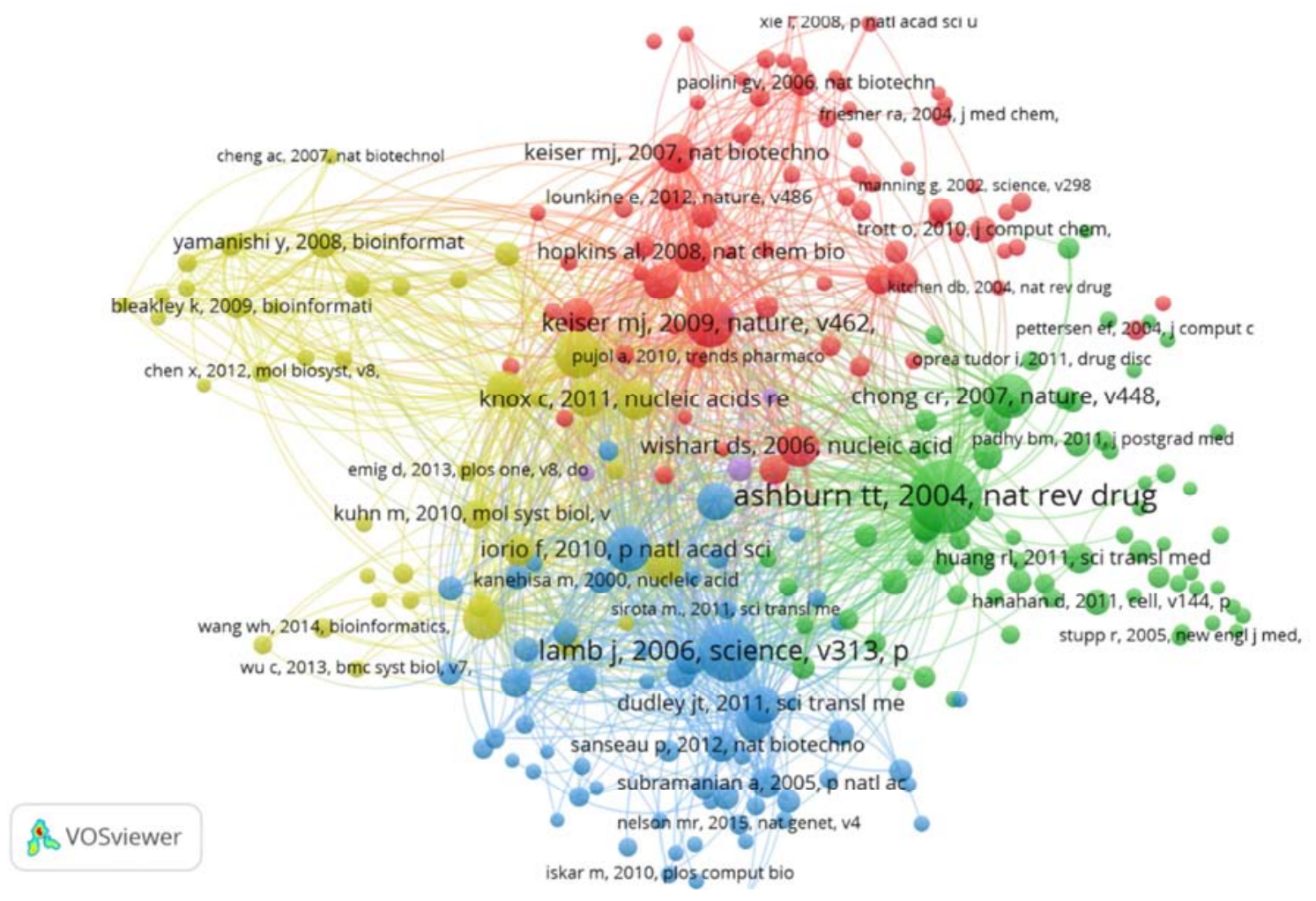

Figure 7. Cited reference co-citation network. 
Table 4. Top 10 cited references in DR research.

\begin{tabular}{llll}
\hline First Author & Cited reference & Citations & Article type \\
\hline Ashburn TT & Drug repositioning: identifying and developing new uses for existing drugs. & 454 & review \\
Lamb J & The Connectivity Map: using gene-expression signatures to connect small molecules, genes, and disease. & 298 & Article \\
Keiser MJ & Predicting new molecular targets for known drugs. & 206 & Article \\
Campillos M & Drug target identification using side-effect similarity. & 190 & Article \\
Chong CR & New uses for old drugs. & 176 & Commentary \\
Iorio F & Discovery of drug mode of action and drug repositioning from transcriptional responses. & 169 & Article \\
Gottlieb A & Predict: a method for inferring novel drug indications with application to personalized medicine. & 142 & Article \\
Knox C & DrugBank 3.0: a comprehensive resource for 'omics' research on drugs. & 141 & review \\
Wishart DS & DrugBank: a comprehensive resource for in silico drug discovery and exploration & 137 & review \\
Keiser MJ & Relating protein pharmacology by ligand chemistry. & 129 & Article \\
\hline
\end{tabular}

Table 5. The top 20 co-occurrence keywords.

\begin{tabular}{llllllll}
\hline Rank & Keyword & Counts & Centrality & Rank & Keyword & Counts & Centrality \\
\hline 1 & drug repurposing & 324 & 0.17 & 11 & breast cancer & 33 & 0.05 \\
2 & drug repositioning & 316 & 0.18 & 12 & inflammation & 32 & 0.06 \\
3 & repurposing & 98 & 0.14 & 13 & virtual screening & 30 & 0.11 \\
4 & cancer & 91 & 0.30 & 14 & clinical trail & 28 & 0.05 \\
5 & drug discovery & 89 & 0.16 & 15 & bioinformatics & 28 & 0.00 \\
6 & apoptosis & 46 & 0.12 & 16 & tuberculosis & 27 & 0.05 \\
7 & alzheimer's disease & 43 & 0.04 & 17 & systems biology & 27 & 0.10 \\
8 & metformin & 38 & 0.10 & 18 & machine learning & 25 & 0.04 \\
9 & polypharmacology & 35 & 0.11 & 19 & drug development & 24 & 0.08 \\
10 & repositioning & 34 & 0.17 & 20 & statin & 24 & 0.06 \\
\hline
\end{tabular}

Table 6. Top 16 keywords with the strongest citation bursts.

\begin{tabular}{|c|c|c|c|c|c|}
\hline Keyword & Year & Strength & Begin & End & 2000-2018 \\
\hline gene & 2000 & 9.41 & 2003 & 2014 & \\
\hline crystal structure & 2000 & 4.45 & 2005 & 2012 & \\
\hline activation & 2000 & 5.82 & 2007 & 2012 & \\
\hline binding & 2000 & 5.52 & 2008 & 2011 & \\
\hline pharmacology & 2000 & 11.82 & 2009 & 2014 & \\
\hline target & 2000 & 10.04 & 2009 & 2013 & \\
\hline chemotherapy & 2000 & 4.96 & 2009 & 2011 & \\
\hline connectivity map & 2000 & 7.39 & 2010 & 2013 & \\
\hline database & 2000 & 12.06 & 2011 & 2014 & \\
\hline design & 2000 & 6.81 & 2011 & 2012 & \\
\hline in vivo & 2000 & 8.02 & 2012 & 2014 & \\
\hline prediction & 2000 & 11.47 & 2013 & 2014 & . \\
\hline protein & 2000 & 7.03 & 2013 & 2015 & \\
\hline inhibition & 2000 & 5.41 & 2013 & 2016 & \\
\hline pathway & 2000 & 17.33 & 2016 & 2018 & \\
\hline apoptosis & 2000 & 17.32 & 2016 & 2018 & 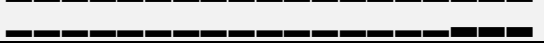 \\
\hline
\end{tabular}

\subsection{Research Hotspots and Emerging Trends of DR}

Here, CiteSpace 5.4 was employed to construct keywords co-occurrence network and to detect strongest citation burst keywords. The centrality of a node was used to quantify the importance of the node's position in a network [14-15]. In CiteSpace 5.4, a node with a centrality of more than 0.1 is called a key node (pivotal point). Keywords with high frequency and centrality represent the research hotspots in a research field to certain extent. Among the top 20 keywords listed in Table 5, five keywords (not including the search word in this study) with a centrality of more than 0.1 were identified as research hotspots in DR study. For instance, cancer (91 counts, 0.30 ), drug discovery ( 89 counts, 0.16 ), apoptosis (46 counts, 0.12), polypharmacology (35 counts, 0.11 ) and virtual screening (30 counts, 0.11 ).

In Table 6, blue lines represent time intervals, while the highlighted red lines represent the periods of burst keywords, indicating the duration of the burst. It can be seen from Table 6 that, before 2009, keywords with the strongest citation bursts were focused on basic research, like "gene", "crystal structure", "pharmacology" and "target". Through basic research, scientists could have a better understanding of mechanisms involved in disease pathogenesis, providing insights into the reuse of an existing drug [16]. After 2010, the strongest citation burst keywords in terms of strategies, such as "connectivity map (cMap)", "database", "pathway" and "apoptosis", accounted for large proportions.

\section{Discussion}

To our knowledge, this was the first application of bibliometric quantitatively and qualitatively methods to 
analyze the DR related publications from WoSCC. The annual number of publications continually increased over time. A dramatic acceleration was appeared in 2010. There were several reasons explained for the sharp growth. First, with the advent and development of high-performance computing, large-scale screening technologies and big data analytics approaches, drug rediscovery was not a serendipitous endeavor [17]. Second, funds support raised for drug repurposing research projects. For example, the National Center for Advancing Translational Sciences (NCATS) and Cures within Reach (CWR) granted most funding for drug repurposing projects [18].

The IF is the indication of a journal influence within its field. Of the top 15 active journals, 4 journals had an IF $>5.0$ (172 articles, 5.34\%); 7 journals had an3.0 $<\mathrm{IF}<5.0(279$ articles, 8.66\%); 4 journals had an IF $<3.0(198$ articles, $6.15 \%$ ). Comparing the rate of high-IF journals to that of all journals, DR related articles were relatively published in high-IF journals.

Co-authorship analysis was employed to evaluate the relationships in terms of authors, countries and institutions. Collaboration level is an index to estimate the research status in a specific area [19]. The USA, which devoted the largest number of articles, article contribution institutions and the most extensive cooperation with other countries, was the most leading country in DR research. Additionally, China was the only developing country in the top three productive country list, which indicating its great effort and progress in medicine and life sciences during the past few decades. However, the rank of publication number of China did not match that of the citations, which to some degree indicated the article quality remained to be improved. For example, the publication number in DR research from the Chinese Academy of Sciences ranked first, while the citations of these articles only ranked 14th. Furthermore, the author's partnership is mainly limited to national authors and the international cooperation remains to be strengthened. Drug discovery is a systematic project and requires extensive cooperation worldwide.

Co-citation analysis could be used to study overall the structure and characteristics of academic journals network [20]. The top 10 most cited articles covering reviews on DR and repurposing strategies, were the intellectual bases in DR research. Keywords reflect the topic and content of an article, providing a reasonable depiction of research hotspots, whereas burst keywords reveal new research frontiers [14]. High frequency keywords, such as cancer, drug discovery, apoptosis, polypharmacology and virtual screening were the research hotspots in this field. Major repurposing strategies can be classified into computational approaches and experimental approaches [21]. The widely used computational approaches were drug-centric approaches [22], target-based approaches [23], knowledge-based methods [24], pathway- or network-based methods [25] and signaturebased approaches [26]. Experimental repurposing strategies were categorized into binding assays [27] and phenotypic screening approaches [28]. In summary, computation-based methods are becoming the mainstream of systematic DR research. A comprehensive application of those repurposing methods will enhance its feasibility in identifying novel indications for old drugs.

\section{Conclusion}

From 2000 to 2018, the number of DR related publications increased year by year. PLoS One published the highest number of publications, followed by Scientific Reports, Oncotarget, Antimicrobial Agents and Chemotherapy and Drug Discovery Today. The USA, which devoted the largest number of articles and the most extensive cooperation with other countries, was the most leading country in this field. Talevi A was the most productive authors and Ashburn TT was the most cited author. The hotspots of DR research were "cancer", "drug discovery", "apoptosis", "polypharmacology" and "virtual screening". In summary, this study gives investigators the landscape of DR research from the perspective of bibliometrics.

\section{Conflict of Interest}

The authors declare that they have no conflict of interest.

\section{Acknowledgements}

This work was supported by the Hubei Province health and family planning scientific research project (No. WJ2018H0167).

\section{References}

[1] Simsek M, Meijer B, van Bodegraven AA, de Boer NKH, Mulder CJJ. Finding hidden treasures in old drugs: the challenges and importance of licensing generics. Drug Discov Today 2018; 23: 17-21.

[2] Jin G, Wong ST. Toward better drug repositioning: prioritizing and integrating existing methods into efficient pipelines. Drug Discov Today 2014; 19: 637-644.

[3] Ashburn TT, Thor KB. Drug repositioning: identifying and developing new uses for existing drugs. Nat Rev Drug Discov 2004; 3: 673-683.

[4] Baker NC, Ekins S, Williams AJ, Tropsha A. A bibliometric review of drug repurposing. Drug Discov Today 2018;23:661672 .

[5] Goldstein I, Lue TF, Padma-Nathan H, Rosen RC, Steers WD, Wicker PA. Oral sildenafil in the treatment of erectile dysfunction. Sildenafil Study Group. N Engl J Med 1998; 338: 1397-1404.

[6] Hou D, Bi X, Mao Z, Fan Y, Hu X, Li X. Biomaterials research of China from 2013 to 2017 based on bibliometrics and visualization analysis. PeerJ 2019; 7: e6859.

[7] Liu H, Yu Z, Chen C, Hong R, Jin K, Yang C. Visualization and bibliometric analysis of research trends on human fatigue assessment. J Med Syst 2018; 42: 179. 
[8] Popkirov S, Jungilligens J, Schlegel U, Wellmer J. Research on dissociative seizures: a bibliometric analysis and visualization of the scientific landscape. Epilepsy Behav 2018; 83: $162-167$.

[9] Zou L, Sun L. Global diabetic kidney disease research from 2000 to 2017. Medicine 2019; 98: e14394.

[10] Zou X, Yue WL, Vu HL. Visualization and analysis of mapping knowledge domain of road safety studies. Accid Anal Prev 2018; 118: 131-145.

[11] Zyoud SH, Smale S, Waring WS, Sweileh WM, Al-Jabi SW. Global research trends in microbiome-gut-brain axis during 2009-2018: a bibliometric and visualized study. BMC Gastroenterol 2019; 19: 158.

[12] Tahamtan I, Safipour Afshar A, Ahamdzadeh K. Factors affecting number of citations: a comprehensive review of the literature. Scientometrics 2016; 107: 1195-1225.

[13] Osareh F. Bibliometncs, citation analysis and co-citation analysis: a review of literature I. Libri. 1996; 49: 149-158.

[14] Chen C. CiteSpace II:detecting and visualizing emerging trends and transient patterns in scientific literature. J Am Soc Inf Sci Technol 2006; 57: 359-377.

[15] Girvan M, Newman MEJ. Community structure in social and biological networks. Proc Natl Acad Sci USA 2002; 99: 78217826.

[16] Parvathaneni V, Kulkarni NS, Muth A, Gupta V. Drug repurposing: a promising tool to accelerate the drug discovery process. Drug Discov Today 2019; 24: 2076-2085.

[17] Keiser MJ, Setola V, Irwin JJ, Laggner C, Abbas AI, Hufeisen $\mathrm{SJ}$, et al. Predicting new molecular targets for known drugs. Nature 2009; 462: 175-181.

[18] Polamreddy P, Gattu N. The drug repurposing landscape from 2012 to 2017: evolution, challenges, and possible solutions. Drug Discov Today 2019; 24:789-795.
[19] Reyes-Gonzalez L, Gonzalez-Brambila CN, Veloso F. Using co-authorship and citation analysis to identify research groups: a new way to assess performance. Scientometrics 2016; 108: 1171-1191.

[20] Small H. Co-citation in the scientific literature: a new measure of the relationship between two documents. J Am Soc Inf Sci 1973; 24: 265-269.

[21] Pushpakom S, Iorio F, Eyers PA, Escott KJ, Hopper S, Wells $\mathrm{A}$, et al. Drug repurposing: progress, challenges and recommendations. Nat Rev Drug Discov 2019; 18: 41-58.

[22] Reddy AS, Zhang S. Polypharmacology: drug discovery for the future. Expert Rev Clin Pharmacol 2013; 6: 41-47.

[23] Sawada R, Iwata H, Mizutani S, Yamanishi Y. Target-based drug repositioning using large-scale chemical-protein interactome data. J Chem Inf Model 2015; 55: 2717-2730.

[24] Hafeez BB, Ganju A, Sikander M, Kashyap VK, Hafeez ZB, Chauhan $\mathrm{N}$, et al. Ormeloxifene suppresses prostate tumor growth and metastatic phenotypes via inhibition of oncogenic beta-catenin signaling and EMT progression. Mol Cancer Ther 2017; 16: 2267-2280.

[25] Iorio F, Saez-Rodriguez J, di Bernardo D. Network based elucidation of drug response: from modulators to targets. BMC Syst Biol 2013; 7: 139.

[26] Wu H, Huang J, Zhong Y, Huang Q. DrugSig: a resource for computational drug repositioning utilizing gene expression signatures. PLoS One 2017; 12: e177743.

[27] Martinez MD, Jafari R, Ignatushchenko M, Seki T, Larsson EA, Dan C, et al. Monitoring drug target engagement in cells and tissues using the cellular thermal shift assay. Science 2013; 341: 84-87.

[28] Zheng W, Thorne N, McKew JC. Phenotypic screens as a renewed approach for drug discovery. Drug Discov Today 2013; 18: 1067-1073. 